\title{
Hyers-Ulam Stability of a System of First Order Linear Recurrences with Constant Coefficients
}

\author{
Bing $X \mathbf{u}^{1}$ and Janusz Brzdę $k^{2}$ \\ ${ }^{1}$ Department of Mathematics, Sichuan University, Chengdu, Sichuan 610064, China \\ ${ }^{2}$ Department of Mathematics, Pedagogical University, Podchorążych 2, 30-084 Kraków, Poland \\ Correspondence should be addressed to Bing Xu; xb4106@sina.com
}

Received 17 November 2014; Accepted 23 January 2015

Academic Editor: Rigoberto Medina

Copyright (C) 2015 B. Xu and J. Brzdęk. This is an open access article distributed under the Creative Commons Attribution License, which permits unrestricted use, distribution, and reproduction in any medium, provided the original work is properly cited.

We study the Hyers-Ulam stability in a Banach space $X$ of the system of first order linear difference equations of the form $\mathbf{x}_{n+1}=$ $A \mathbf{x}_{n}+\mathbf{d}_{n}$ for $n \in \mathbb{N}_{0}$ (nonnegative integers), where $A$ is a given $r \times r$ matrix with real or complex coefficients, respectively, and $\left(\mathbf{d}_{n}\right)_{n \in \mathbb{N}_{0}}$ is a fixed sequence in $X^{r}$. That is, we investigate the sequences $\left(\mathbf{y}_{n}\right)_{n \in \mathbb{N}_{0}}$ in $X^{r}$ such that $\delta:=\sup _{n \in \mathbb{N}_{0}}\left\|\mathbf{y}_{n+1}-A \mathbf{y}_{n}-\mathbf{d}_{n}\right\|<\infty$ (with the maximum norm in $X^{r}$ ) and show that, in the case where all the eigenvalues of $A$ are not of modulus 1, there is a positive real constant $c$ (dependent only on $A$ ) such that, for each such a sequence $\left(\mathbf{y}_{n}\right)_{n \in \mathbb{N}_{0}}$, there is a solution $\left(\mathbf{x}_{n}\right)_{n \in \mathbb{N}_{0}}$ of the system with $\sup _{n \in \mathbb{N}_{0}}\left\|\mathbf{y}_{n}-\mathbf{x}_{n}\right\| \leq c \delta$.

\section{Introduction}

The issue of stability of a functional equation can be expressed in the following way. When must a function satisfying an equation approximately (in some sense) be near an exact solution to the equation? It has been motivated by a question raised in 1940 by Ulam, concerning approximate homomorphisms of groups (see $[1,2])$. The first partial answer to Ulam's question (in the case of Cauchy's functional equation in Banach spaces) was given by Hyers in [1]. After that result, a great number of papers on the subject have been published (see, e.g., monographs [3-5], survey articles [6-11], and the references given there), generalizing Ulam's problem and Hyers's theorem in various directions and to other equations (not necessarily functional) (see [12]). In particular, some results have been proved in [13], which concern the stability of linear difference equations of higher order of form (1). We describe them as follows.

Let $T$ be either $\mathbb{N}_{0}$ (the set of nonnegative integers) or $\mathbb{Z}$ (the set of integers), let $\mathbb{K}$ be either the field of reals $\mathbb{R}$ or the field of complex numbers $\mathbb{C}$, let $p \in \mathbb{N}$ (the set of positive integers), let $a_{1}, \ldots, a_{p} \in \mathbb{K}$ be fixed, and let $\left(b_{n}\right)_{n \in T}$ be a given sequence in a Banach space $X$ over $\mathbb{K}$. The investigation of the Hyers-Ulam stability in $X$ of the difference equation

$$
x_{n+p}=a_{1} x_{n+p-1}+\cdots+a_{p} x_{n}+b_{n}, \quad n \in T,
$$

actually means a study of the sequences $\left(y_{n}\right)_{n \in T}$ in $X$, satisfying the condition

$$
\delta:=\sup _{n \in T}\left\|y_{n+p}-a_{1} y_{n+p-1}-\cdots-a_{p} y_{n}-b_{n}\right\|<\infty .
$$

Let $\mathbb{S}=\{z \in \mathbb{C}:|z|=1\}$ and $t_{1}, \ldots, t_{p} \in \mathbb{C}$ denote the roots of the characteristic equation of (1), which has the following form: $z^{p}=a_{1} z^{p-1}+\cdots+a_{p}$. The following two theorems have been proved in [13] (see also [14, 15]).

Theorem 1. Let $\delta>0$ and $t_{1}, \ldots, t_{p} \in \mathbb{C} \backslash \mathbb{S}$. Suppose that $\left(y_{n}\right)_{n \in T}$ is a sequence in $X$ such that (2) holds. Then, there exists a sequence $\left(x_{n}\right)_{n \in T}$ in $X$ satisfying (1) such that

$$
\left\|y_{n}-x_{n}\right\| \leq \frac{\delta}{|1-| t_{1}|| \cdots|1-| t_{p}||}, \quad n \in T .
$$


Moreover,

(a) $\left(x_{n}\right)_{n \in T}$ is unique if and only if $\left|t_{i}\right|>1$ for $i=1, \ldots, p$ or $T=\mathbb{Z}$;

(b) if $\left|t_{i}\right|>1$ for $i \in\{1, \ldots, p\}$ or $T=\mathbb{Z}$, then $\left(x_{n}\right)_{n \in T}$ is the unique sequence in $X$ such that (1) holds and $\sup _{n \in T}\left\|x_{n}-y_{n}\right\|<\infty$;

(c) if $T=\mathbb{N}_{0}$ and $\left|t_{i}\right|<1$ for some $i \in\{1, \ldots, p\}$, then the cardinality of the set of all sequences $\left(x_{n}\right)_{n \in T}$ in $X$, satisfying (1) and (3), equals the cardinality of $X$.

Theorem 2. Let $\left|t_{j}\right|=1$ for some $j \in\{1, \ldots, p\}$. Then, for any $\delta>0$, there exists a sequence $\left(y_{n}\right)_{n \in T}$ in $X$, satisfying inequality (2), such that, for every sequence $\left(x_{n}\right)_{n \in T}$ in $X$, fulfilling recurrence (1),

$$
\sup _{n \in T}\left\|y_{n}-x_{n}\right\|=\infty
$$

Moreover, if $t_{1}, \ldots, t_{p} \in \mathbb{K}$ or there is a bounded sequence $\left(x_{n}\right)_{n \in T}$ in $X$ fulfilling (1), then $\left(y_{n}\right)_{n \in T}$ can be chosen unbounded.

We somehow complement those results in this paper by the study of the Hyers-Ulam stability of the following system of first order linear difference equations in $X$ with constant coefficients $a_{i j} \in \mathbb{K}, i, j=1, \ldots, r(r \in \mathbb{N}$ is fixed):

$$
\begin{aligned}
x_{n+1}^{1} & =a_{11} x_{n}^{1}+a_{12} x_{n}^{2}+\cdots+a_{1 r} x_{n}^{r}+d_{n}^{1}, \\
x_{n+1}^{2}= & a_{21} x_{n}^{1}+a_{22} x_{n}^{2}+\cdots+a_{2 r} x_{n}^{r}+d_{n}^{2}, \\
& \vdots \\
x_{n+1}^{r} & =a_{r 1} x_{n}^{1}+a_{r 2} x_{n}^{2}+\cdots+a_{r r} x_{n}^{r}+d_{n}^{r},
\end{aligned}
$$

for all $n \in \mathbb{N}_{0}$, where $d_{n}^{1}, \ldots, d_{n}^{r} \in X$ for $n \in \mathbb{N}_{0}$ are given. If we write

$$
\begin{gathered}
A=\left[\begin{array}{cccc}
a_{11} & a_{12} & \cdots & a_{1 r} \\
a_{21} & a_{22} & \cdots & a_{2 r} \\
\vdots & \vdots & \ddots & \vdots \\
a_{r 1} & a_{r 2} & \cdots & a_{r r}
\end{array}\right], \\
\mathbf{x}_{n}=\left[\begin{array}{c}
x_{n}^{1} \\
x_{n}^{2} \\
\vdots \\
x_{n}^{r}
\end{array}\right],
\end{gathered}
$$

then (5) can be expressed in the following simple form:

$$
\mathbf{x}_{n+1}=A \mathbf{x}_{n}+\mathbf{d}_{n}, \quad n \in \mathbb{N}_{0} .
$$

To simplify the notations, we consider $\mathbf{x}_{n}$ and $\mathbf{d}_{n}$ to be elements of $X^{r}$, when it is convenient (and when this makes no confusion); that is, we identify $\mathbf{x}_{n}$ with $\left(x_{n}^{1}, \ldots, x_{n}^{r}\right)$ and $\mathbf{d}_{n}$ with $\left(d_{n}^{1}, \ldots, d_{n}^{r}\right)$.

Our results correspond, in particular, not only to the outcomes in [13], but also to those in $[14,15]$, where similar problems have been studied for $r=1$.

\section{Some Auxiliary Results}

By an elementary induction on $n$, we obtain the following simple observation.

Lemma 3. If a sequence $\left(\mathbf{x}_{n}\right)_{n \in \mathbb{N}_{0}}$ in $X^{r}$ satisfies (7), then

$$
\mathbf{x}_{n}=A^{n} \mathbf{x}_{0}+\sum_{k=1}^{n} A^{n-k} \mathbf{d}_{k-1}, \quad n \in \mathbb{N}
$$

In this paper,

$$
C_{j}^{n}:=\left(\begin{array}{c}
n \\
j
\end{array}\right)=\frac{n !}{(n-j) ! j !}, \quad n, j \in \mathbb{N}_{0}, n \geq j,
$$

denote the binomial coefficients and $C_{j}^{n}:=0$ for $j>n$. The subsequent formula is well known:

$$
\sum_{n=0}^{\infty} C_{n}^{n+j} w^{n}=\frac{1}{(1-w)^{j+1}}, \quad w \in[0,1), \quad j \in \mathbb{N}_{0} .
$$

Also, replacing $w$ by $1 / x$, we easily obtain that

$$
\sum_{n=0}^{\infty} C_{n}^{n+j} x^{-(n+j+1)}=\frac{1}{(x-1)^{j+1}}, \quad x \in(1, \infty), j \in \mathbb{N}_{0}
$$

Further, write

$$
\begin{gathered}
\|A\|:=\max _{1 \leq i \leq r} \sum_{j=1}^{r}\left|a_{i j}\right|, \\
\|\mathbf{x}\|:=\max _{1 \leq i \leq r}\left\|x_{i}\right\|, \quad \mathbf{x}=\left(x_{1}, \ldots, x_{r}\right) \in X^{r} .
\end{gathered}
$$

Then, $\left(X^{r},\|\cdot\|\right)$ is a Banach space and we have the following result, which will be useful in the proof of the main theorem.

Theorem 4. Let $J_{\lambda, r}$ be a Jordan matrix of the form

$$
J_{\lambda, r}=\left[\begin{array}{cccccc}
\lambda & 1 & 0 & \cdots & 0 & 0 \\
0 & \lambda & 1 & \cdots & 0 & 0 \\
\vdots & \vdots & \vdots & \ddots & \vdots & \vdots \\
0 & 0 & 0 & \cdots & \lambda & 1 \\
0 & 0 & 0 & \cdots & 0 & \lambda
\end{array}\right]_{r \times r},
$$

with some $\lambda \in \mathbb{C} \backslash \mathbb{S}$. If a sequence $\left(\mathbf{y}_{n}\right)_{n \in \mathbb{N}_{0}}$ in $X^{r}$ satisfies

$$
\delta:=\sup _{n \in \mathbb{N}_{0}}\left\|\mathbf{y}_{n+1}-J_{\lambda, r} \mathbf{y}_{n}-\mathbf{b}_{n}\right\|<\infty
$$

then there exists a sequence $\left(\mathbf{x}_{n}\right)_{n \in \mathbb{N}_{0}}$ in $X^{r}$ such that

$$
\begin{gathered}
\mathbf{x}_{n+1}=J_{\lambda, r} \mathbf{x}_{n}+\mathbf{b}_{n}, \quad n \in \mathbb{N}_{0}, \\
\sup _{n \in \mathbb{N}_{0}}\left\|\mathbf{y}_{n}-\mathbf{x}_{n}\right\| \leq\left.\delta \sum_{j=1}^{r}|1-| \lambda\right|^{-j} .
\end{gathered}
$$


Proof. Let $\mathbf{c}_{n}:=\mathbf{y}_{n+1}-J_{\lambda, r} \mathbf{y}_{n}-\mathbf{b}_{n}$ for $n \in \mathbb{N}_{0}$. Then, by (14), $\left\|\mathbf{c}_{n}\right\| \leq \delta$ for $n \in \mathbb{N}_{0}$ and (see Lemma 3)

$$
\mathbf{y}_{n}=J_{\lambda, r}^{n} \mathbf{y}_{0}+\sum_{k=1}^{n} J_{\lambda, r}^{n-k}\left(\mathbf{b}_{k-1}+\mathbf{c}_{k-1}\right), \quad n \in \mathbb{N} .
$$

Case $1(|\lambda|<1)$. Define the sequence $\left(\mathbf{x}_{n}\right)_{n \in \mathbb{N}_{0}}$ by (15) with $\mathbf{x}_{0}=\mathbf{y}_{0}$. Then,

$$
\left\|\mathbf{y}_{n}-\mathbf{x}_{n}\right\|=\left\|\sum_{k=1}^{n} J_{\lambda, r}^{n-k} \mathbf{c}_{k-1}\right\| \leq \delta \sum_{k=1}^{n}\left\|J_{\lambda, r}^{n-k}\right\|, \quad n \in \mathbb{N} .
$$

It is easy to show by induction on $n$ that $J_{\lambda, r}^{n}$ is an upper (right) triangular matrix of the form

$$
J_{\lambda, r}^{n}=\left[\begin{array}{cccc}
\lambda^{n} & C_{1}^{n} \lambda^{n-1} & \cdots & C_{r-1}^{n} \lambda^{n-(r-1)} \\
0 & \lambda^{n} & \cdots & C_{r-2}^{n} \lambda^{n-(r-2)} \\
\vdots & \vdots & \ddots & \vdots \\
0 & 0 & \cdots & C_{1}^{n} \lambda^{n-1} \\
0 & 0 & \cdots & \lambda^{n}
\end{array}\right]_{r \times r}
$$

(it is enough to use the well-known formula $C_{k+1}^{n+1}=C_{k}^{n}+C_{k+1}^{n}$ ) whence we derive that $\left\|J_{\lambda, r}^{n}\right\|=\sum_{j=0}^{r-1} C_{j}^{n}|\lambda|^{n-j}$ for $n \in \mathbb{N}_{0}$.

Since, in view of (10),

$$
\sum_{n=0}^{\infty} C_{j}^{n}|\lambda|^{n-j}=\frac{1}{(1-|\lambda|)^{j+1}}, \quad j=0, \ldots, r
$$

we have

$$
\begin{aligned}
\sum_{n=0}^{\infty}\left\|J_{\lambda, r}^{n}\right\| & =\sum_{n=0}^{\infty} \sum_{j=0}^{r-1} C_{j}^{n}|\lambda|^{n-j}=\sum_{j=0}^{r-1} \sum_{n=0}^{\infty} C_{j}^{n}|\lambda|^{n-j} \\
& =\sum_{j=1}^{r} \frac{1}{(1-|\lambda|)^{j}}
\end{aligned}
$$

and, by (18),

$$
\left\|\mathbf{y}_{n}-\mathbf{x}_{n}\right\| \leq \sum_{k=0}^{\infty}\left\|J_{\lambda, r}^{k}\right\| \delta=\delta \sum_{j=1}^{r} \frac{1}{(1-|\lambda|)^{j}}, \quad n \in \mathbb{N}_{0} .
$$

That is, inequality (16) holds.

Case $2(|\lambda|>1)$. Since $J_{\lambda, r}^{-1}$ is an upper triangular matrix of the form

$$
J_{\lambda, r}^{-1}=\left[\begin{array}{cccc}
\lambda^{-1} & \frac{-1}{\lambda^{2}} & \cdots & \frac{(-1)^{r-1}}{\lambda^{r}} \\
0 & \lambda^{-1} & \cdots & \frac{(-1)^{r-2}}{\lambda^{r-1}} \\
\vdots & \vdots & \ddots & \vdots \\
0 & 0 & \cdots & \frac{-1}{\lambda^{2}} \\
0 & 0 & \cdots & \lambda^{-1}
\end{array}\right]_{r \times r}
$$

it is easy to check that $J_{\lambda, r}^{-n}$ is also an upper triangular matrix for each $n \in \mathbb{N}$ and has the form

$$
J_{\lambda, r}^{-n}=\left[\begin{array}{cccc}
\lambda^{-n} & \frac{-C_{n-1}^{n}}{\lambda^{n+1}} & \cdots & \frac{(-1)^{r-1} C_{n-1}^{n+r-2}}{\lambda^{n+r-1}} \\
0 & \lambda^{-n} & \cdots & \frac{(-1)^{r-2} C_{n-1}^{n+r-3}}{\lambda^{n+r-2}} \\
\vdots & \vdots & \ddots & \vdots \\
0 & 0 & \cdots & \frac{-C_{n-1}^{n}}{\lambda^{n+1}} \\
0 & 0 & \cdots & \lambda^{-n}
\end{array}\right]_{r \times r} .
$$

Hence,

$$
\left\|J_{\lambda, r}^{-n}\right\|=\sum_{j=0}^{r-1} C_{n-1}^{n-1+j}|\lambda|^{-(n+j)}, \quad n \in \mathbb{N} .
$$

Consequently, in view of (11),

$$
\begin{aligned}
\sum_{n=1}^{\infty}\left\|J_{\lambda, r}^{-n}\right\| & =\sum_{n=0}^{\infty} \sum_{j=0}^{r-1} C_{n}^{n+j}|\lambda|^{-(n+j+1)} \\
& =\sum_{j=0}^{r-1} \sum_{n=0}^{\infty} C_{n}^{n+j}|\lambda|^{-(n+j+1)} \\
& =\sum_{j=1}^{r} \frac{1}{(|\lambda|-1)^{j}} .
\end{aligned}
$$

Taking into account that $\left\|J_{\lambda, r}^{-k} \mathbf{c}_{k-1}\right\| \leq\left\|J_{\lambda, r}^{-k}\right\|\left\|\mathbf{c}_{k-1}\right\| \leq \delta\left\|J_{\lambda, r}^{-k}\right\|$ for $k \in \mathbb{N}$, we deduce that the series $\sum_{k=1}^{\infty} J_{\lambda, r}^{-k} \mathbf{c}_{k-1}$ is convergent. Take

$$
\mathbf{s}:=\sum_{k=1}^{\infty} J_{\lambda, r}^{-k} \mathbf{c}_{k-1}
$$

and define $\left(\mathbf{x}_{n}\right)_{n \in \mathbb{N}_{0}}$ by (15) with $\mathbf{x}_{0}=\mathbf{y}_{0}+\mathbf{s}$. Then, (see Lemma 3)

$$
\mathbf{x}_{n}=J_{\lambda, r}^{n}\left(\mathbf{y}_{0}+\mathbf{s}\right)+\sum_{k=1}^{n} J_{\lambda, r}^{n-k} \mathbf{b}_{k-1}, \quad n \in \mathbb{N} .
$$

Next, by (26), for every $n \in \mathbb{N}$, we have

$$
\begin{aligned}
\left\|\mathbf{y}_{n}-\mathbf{x}_{n}\right\| & =\left\|\sum_{k=1}^{n} J_{\lambda, r}^{n-k} \mathbf{c}_{k-1}-J_{\lambda, r}^{n} \mathbf{s}\right\| \\
& =\left\|\sum_{k=1}^{n} J_{\lambda, r}^{n-k} \mathbf{c}_{k-1}-J_{\lambda, r}^{n} \sum_{k=1}^{\infty} J_{\lambda, r}^{-k} \mathbf{c}_{k-1}\right\| \\
& =\left\|\sum_{k=1}^{n} J_{\lambda, r}^{n-k} \mathbf{c}_{k-1}-\sum_{k=1}^{\infty} J_{\lambda, r}^{n-k} \mathbf{c}_{k-1}\right\| \\
& =\left\|\sum_{k=n+1}^{\infty} J_{\lambda, r}^{n-k} \mathbf{c}_{k-1}\right\|,
\end{aligned}
$$

whence

$$
\left\|\mathbf{y}_{n}-\mathbf{x}_{n}\right\| \leq \sum_{k=1}^{\infty}\left\|J_{\lambda, r}^{-k}\right\| \delta=\delta \sum_{j=1}^{r} \frac{1}{(|\lambda|-1)^{j}}, \quad n \in \mathbb{N}_{0} .
$$




\section{The Main Result}

Let $\lambda_{1}, \ldots, \lambda_{m}$ be the eigenvalues of $A$ with multiplicities $r_{1}, \ldots, r_{m}$, respectively. There exists a nonsingular matrix $Q$ in $\mathbb{C}^{r \times r}$ with $A=Q J Q^{-1}$, where

$$
\begin{gathered}
J=J_{\lambda_{1}, r_{1}} \oplus \cdots \oplus J_{\lambda_{m}, r_{m}}, \\
J_{\lambda_{j}, r_{j}}=\left[\begin{array}{cccccc}
\lambda_{j} & 1 & 0 & \cdots & 0 & 0 \\
0 & \lambda_{j} & 1 & \cdots & 0 & 0 \\
\vdots & \vdots & \vdots & \ddots & \vdots & \vdots \\
0 & 0 & 0 & \cdots & \lambda_{j} & 1 \\
0 & 0 & 0 & \cdots & 0 & \lambda_{j}
\end{array}\right]_{r_{j} \times r_{j}}, j=1, \ldots, m .
\end{gathered}
$$

The next theorem is the main result of this paper.

Theorem 5. Assume that $\lambda_{j} \in \mathbb{C} \backslash \mathbb{S}$ for $j=1, \ldots, m$. For any sequence $\left(\mathbf{z}_{n}\right)_{n \in \mathbb{N}_{0}}$ in $X^{r}$, satisfying

$$
\delta:=\sup _{n \in \mathbb{N}_{0}}\left\|\mathbf{z}_{n+1}-A \mathbf{z}_{n}-\mathbf{d}_{n}\right\|<\infty
$$

there exists a sequence $\left(\mathbf{x}_{n}\right)_{n \in \mathbb{N}_{0}}$ in $X^{r}$ such that

$$
\begin{gathered}
\mathbf{x}_{n+1}=A \mathbf{x}_{n}+\mathbf{d}_{n}, \quad n \in \mathbb{N}_{0}, \\
\sup _{n \in \mathbb{N}_{0}}\left\|\mathbf{z}_{n}-\mathbf{x}_{n}\right\| \leq \delta\|Q\|\left\|Q^{-1}\right\| \max _{j=1, \ldots, m} \sum_{k=1}^{r_{j}} \frac{1}{|1-| \lambda_{j} \|^{k}} .
\end{gathered}
$$

Proof. Let $\left(\mathbf{z}_{n}\right)_{n \in \mathbb{N}_{0}}$ be a sequence in $X^{r}$ such that (32) holds.

First, consider the case where $\mathbb{K}=\mathbb{C}$. Write $\mathbf{y}_{n}:=Q^{-1} \mathbf{z}_{n}$ and $\mathbf{b}_{n}:=Q^{-1} \mathbf{d}_{n}$ for $n \in \mathbb{N}_{0}$. Then,

$$
\begin{aligned}
\left\|\mathbf{y}_{n+1}-J \mathbf{y}_{n}-\mathbf{b}_{n}\right\| & \leq\left\|Q^{-1}\right\|\left\|\mathbf{z}_{n+1}-A \mathbf{z}_{n}-\mathbf{d}_{n}\right\| \\
& \leq\left\|Q^{-1}\right\| \delta=: \delta_{0}, \quad n \in \mathbb{N}_{0} .
\end{aligned}
$$

Define projections $p_{j}: X^{r} \rightarrow X^{r_{j}}($ for $j=1, \ldots, m)$ by

$$
\begin{aligned}
& p_{1}\left(w_{1}, \ldots, w_{r}\right):=\left(w_{1}, \ldots, w_{r_{1}}\right), \\
& p_{2}\left(w_{1}, \ldots, w_{r}\right):=\left(w_{r_{1}+1}, \ldots, w_{r_{1}+r_{2}}\right), \\
& \vdots \\
& p_{m-1}\left(w_{1}, \ldots, w_{r}\right):=\left(w_{r_{1}+\cdots+r_{m-2}+1}, \ldots, w_{r_{1}+\cdots+r_{m-1}}\right), \\
& p_{m}\left(w_{1}, \ldots, w_{r}\right):=\left(w_{r_{1}+\cdots+r_{m-1}+1}, \ldots, w_{r_{1}+\cdots+r_{m}}\right)
\end{aligned}
$$

for $\left(w_{1}, \ldots, w_{r}\right) \in X^{r}$. For simplicity, we write $\mathbf{y}_{n}=$ $\left(p_{1}\left(\mathbf{y}_{n}\right), \ldots, p_{m}\left(\mathbf{y}_{n}\right)\right)$ and $\mathbf{b}_{n}=\left(p_{1}\left(\mathbf{b}_{n}\right), \ldots, p_{m}\left(\mathbf{b}_{n}\right)\right)$ for $n \in$ $\mathbb{N}_{0}$. It is easily seen that (in analogous notation)

$$
\begin{gathered}
J \mathbf{y}_{n}=\left(J_{\lambda_{1}, r_{1}} p_{1}\left(\mathbf{y}_{n}\right), \ldots, J_{\lambda_{m}, r_{m}} p_{m}\left(\mathbf{y}_{n}\right)\right), \quad n \in \mathbb{N}_{0}, \\
\left\|p_{j}\left(\mathbf{y}_{n+1}\right)-J_{\lambda_{j}, r_{j}} p_{j}\left(\mathbf{y}_{n}\right)-p_{j}\left(\mathbf{b}_{n}\right)\right\| \\
\leq\left\|\mathbf{y}_{n+1}-J \mathbf{y}_{n}-\mathbf{b}_{n}\right\| \leq \delta_{0}, \\
n \in \mathbb{N}_{0}, \quad j=1, \ldots, m .
\end{gathered}
$$

According to Theorem 4 (applied for each $j \in\{1, \ldots, m\}$, separately), there exists a sequence $\left(\mathbf{u}_{n}\right)_{n \in \mathbb{N}_{0}}$ in $X^{r}$ such that

$$
\begin{gathered}
p_{j}\left(\mathbf{u}_{n+1}\right)=J_{\lambda_{j}, r_{j}} p_{j}\left(\mathbf{u}_{n}\right)+p_{j}\left(\mathbf{b}_{n}\right), \quad n \in \mathbb{N}_{0}, \\
\left\|p_{j}\left(\mathbf{u}_{n}\right)-p_{j}\left(\mathbf{y}_{n}\right)\right\| \leq \delta_{0} \sum_{k=1}^{r_{j}} \frac{1}{|1-| \lambda_{j}||^{k}}, \\
n \in \mathbb{N}_{0}, \quad j=1, \ldots, m .
\end{gathered}
$$

Clearly,

$$
\begin{gathered}
\mathbf{u}_{n+1}=J \mathbf{u}_{n}+\mathbf{b}_{n}, \quad n \in \mathbb{N}_{0}, \\
\left\|\mathbf{y}_{n}-\mathbf{u}_{n}\right\| \leq \delta_{0} \max \left\{\sum_{k=1}^{r_{j}} \frac{1}{|1-| \lambda_{j}||^{k}}: j=1, \ldots, m\right\}, \\
n \in \mathbb{N}_{0} .
\end{gathered}
$$

Let $\mathbf{x}_{n}:=Q \mathbf{u}_{n}$ for $n \in \mathbb{N}_{0}$. Then,

$$
\begin{aligned}
& \mathbf{x}_{n+1}=Q \mathbf{u}_{n+1}=Q J \mathbf{u}_{n}+Q \mathbf{b}_{n}=Q J Q^{-1} \mathbf{x}_{n}+\mathbf{d}_{n}, \quad n \in \mathbb{N}_{0}, \\
&\left\|\mathbf{z}_{n}-\mathbf{x}_{n}\right\|=\left\|Q \mathbf{y}_{n}-Q \mathbf{u}_{n}\right\| \leq\|Q\|\left\|\mathbf{y}_{n}-\mathbf{u}_{n}\right\| \\
& \leq\|Q\| \delta_{0} \max \left\{\sum_{k=1}^{r_{j}} \frac{1}{|1-| \lambda_{j} \|^{k}}: j=1, \ldots, m\right\}, \\
& n \in \mathbb{N}_{0} .
\end{aligned}
$$

Now, consider the case $\mathbb{K}=\mathbb{R}$. Define the linear structure in $\widehat{X}:=X^{2}$ by $(x, y)+(z, w):=(x+z, y+w)$ and $(\alpha+$ $i \beta)(x, y):=(\alpha x-\beta y, \beta x+\alpha y)$ for $x, y, z, w \in X, \alpha, \beta \in \mathbb{R}$. Then, $\widehat{X}$ is a complex Banach space (see, e.g., [16, page 39], [17], or [18, 1.9.6, page 66]), when endowed with the Taylor norm $\|\cdot\|_{T}$ given by

$$
\|(x, y)\|_{T}:=\sup _{0 \leq \theta \leq 2 \pi}\|(\cos \theta) x+(\sin \theta) y\|, \quad x, y \in X .
$$

Note that

$$
\max \{\|x\|,\|y\|\} \leq\|(x, y)\|_{T} \leq\|x\|+\|y\|, \quad x, y \in X .
$$


Define $\widehat{p}_{1}: X^{2} \rightarrow X$ by $\widehat{p}_{1}\left(w_{1}, w_{2}\right):=w_{1}$ for $w_{1}, w_{2} \in X$. Let $\widehat{\mathbf{z}}_{n}:=\left(\mathbf{z}_{n}, 0\right)$ and $\widehat{\mathbf{d}}_{n}:=\left(\mathbf{d}_{n}, 0\right)$ for $n \in \mathbb{N}_{0}$. Then, $\left(\widehat{\mathbf{z}}_{n}\right)_{n \in \mathbb{N}_{0}}$ is a sequence in $\widehat{X}$ and

$$
\left\|\widehat{\mathbf{z}}_{n+1}-A \widehat{\mathbf{z}}_{n}-\widehat{\mathbf{d}}_{n}\right\|_{T}=\left\|\mathbf{z}_{n+1}-A \mathbf{z}_{n}-\mathbf{d}_{n}\right\| \leq \delta, \quad n \in \mathbb{N}_{0} .
$$

So, by the first part of the proof, there is a sequence $\left(\widehat{\mathbf{x}}_{n}\right)_{n \in \mathbb{N}_{0}}$ in $\widehat{X}$ such that

$$
\begin{gathered}
\widehat{\mathbf{x}}_{n+1}=A \widehat{\mathbf{x}}_{n}+\widehat{\mathbf{d}}_{n}, \quad n \in \mathbb{N}_{0}, \\
\sup _{n \in \mathbb{N}_{0}}\left\|\widehat{\mathbf{z}}_{n}-\widehat{\mathbf{x}}_{n}\right\|_{T} \leq \delta\|Q\|\left\|Q^{-1}\right\| \max _{j=1, \ldots, m} \sum_{k=1}^{r_{j}} \frac{1}{\left.|1-| \lambda_{j}\right|^{k}} .
\end{gathered}
$$

Write $\mathbf{x}_{n}:=\widehat{p}_{1}\left(\widehat{\mathbf{x}}_{n}\right)$ for $n \in \mathbb{N}_{0}$. Then, it is easily seen that (33) and (34) are valid (in view of (42)).

Remark 6. The assumption that $\left|\lambda_{j}\right| \neq 1$ for $j=1, \ldots, m$ cannot be omitted in the general case (at least when $r=1$ ), in view of Theorem 2 .

It seems that our method cannot be easily applied to the systems of linear difference equations of higher orders, because it is difficult in such cases to obtain a formula analogous as (8).

Open Problems. There arises a natural question if some results similar to Theorem 2 and statements (a)-(c) of Theorem 1 can be obtained for difference equation (33) with $r>1$ (also with $\mathbb{N}_{0}$ replaced by $\mathbb{Z}$ ).

\section{Conflict of Interests}

The authors declare that there is no conflict of interests regarding the publication of this paper.

\section{Acknowledgment}

Bing Xu is supported by NSFC (China) Grant no. 11101295.

\section{References}

[1] D. H. Hyers, "On the stability of the linear functional equation," Proceedings of the National Academy of Sciences of the United States of America, vol. 27, pp. 222-224, 1941.

[2] S. M. Ulam, A Collection of Mathematical Problems, Interscience Tracts in Pure and Applied Mathematics, Interscience, New York, NY, USA, 1960.

[3] D. H. Hyers, G. Isac, and T. M. Rassias, Stability of Functional Equations in Several Variables, Progress in Nonlinear Differential Equations and Their Applications, Birkhäuser, Boston, Mass, USA, 1998.

[4] S.-M. Jung, Hyers-Ulam-Rassias Stability of Functional Equations in Mathematical Analysis, Hadronic Press, Palm Harbor, Fla, USA, 2001.

[5] S.-M. Jung, Hyers-Ulam-Rassias Stability of Functional Equations in Nonlinear Analysis, Springer, New York, NY, USA, 2011.

[6] R. P. Agarwal, B. Xu, and W. Zhang, "Stability of functional equations in single variable," Journal of Mathematical Analysis and Applications, vol. 288, no. 2, pp. 852-869, 2003.
[7] N. Brillouët-Belluot, J. Brzdęk, and K. Ciepliński, "On some recent developments in Ulam's type stability," Abstract and Applied Analysis, vol. 2012, Article ID 716936, 41 pages, 2012.

[8] J. Brzdęk, K. Ciepliński, and Z. Leśniak, “On Ulam’s type stability of the linear equation and related issues," Discrete Dynamics in Nature and Society, vol. 2014, Article ID 536791, 14 pages, 2014.

[9] G. L. Forti, "Hyers-Ulam stability of functional equations in several variables," Aequationes Mathematicae, vol. 50, no. 1-2, pp. 143-190, 1995.

[10] D. H. Hyers and T. M. Rassias, "Approximate homomorphisms," Aequationes Mathematicae, vol. 44, no. 2-3, pp. 125-153, 1992.

[11] T. M. Rassias, "On the stability of functional equations and a problem of Ulam," Acta Applicandae Mathematicae, vol. 62, no. 1, pp. 23-130, 2000.

[12] S.-M. Jung, "Hyers-Ulam stability of a system of first order linear differential equations with constant coefficients," Journal of Mathematical Analysis and Applications, vol. 320, no. 2, pp. 549-561, 2006.

[13] J. Brzdęk, D. Popa, and B. Xu, "Remarks on stability of linear recurrence of higher order," Applied Mathematics Letters, vol. 23, no. 12, pp. 1459-1463, 2010.

[14] D. Popa, "Hyers-Ulam-Rassias stability of a linear recurrence," Journal of Mathematical Analysis and Applications, vol. 309, no. 2, pp. 591-597, 2005.

[15] D. Popa, "Hyers-Ulam stability of the linear recurrence with constant coefficients," Advances in Difference Equations, vol. 2005, Article ID 407076, 2005.

[16] M. Fabian, P. Habala, P. Hájek, V. Montesinos Santalucía, J. Pelant, and V. Zizler, Functional Analysis and Infinite-Dimensional Geometry, Springer, New York, NY, USA, 2001.

[17] J. Ferrera and G. A. Muñoz, "A characterization of real Hilbert spaces using the Bochnak complexification norm," Archiv der Mathematik, vol. 80, no. 4, pp. 384-392, 2003.

[18] R. V. Kadison and J. R. Ringrose, Fundamentals of the Theory of Operator Algebras, v. I: Elementary Theory, vol. 15 of Graduate Studies in Mathematics, American Mathematical Society, Providence, RI, USA, 1997. 


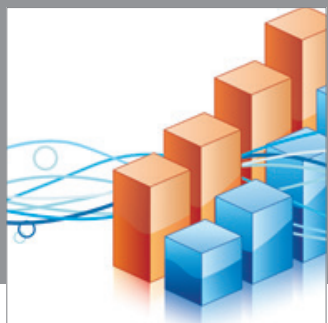

Advances in

Operations Research

mansans

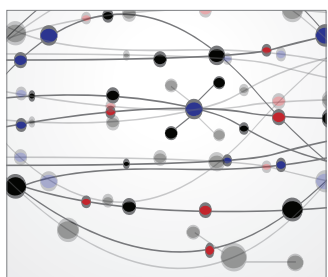

The Scientific World Journal
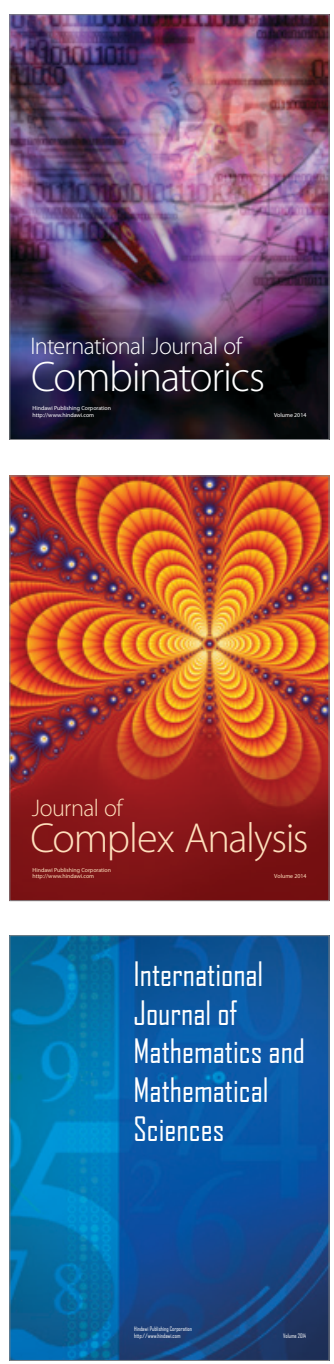
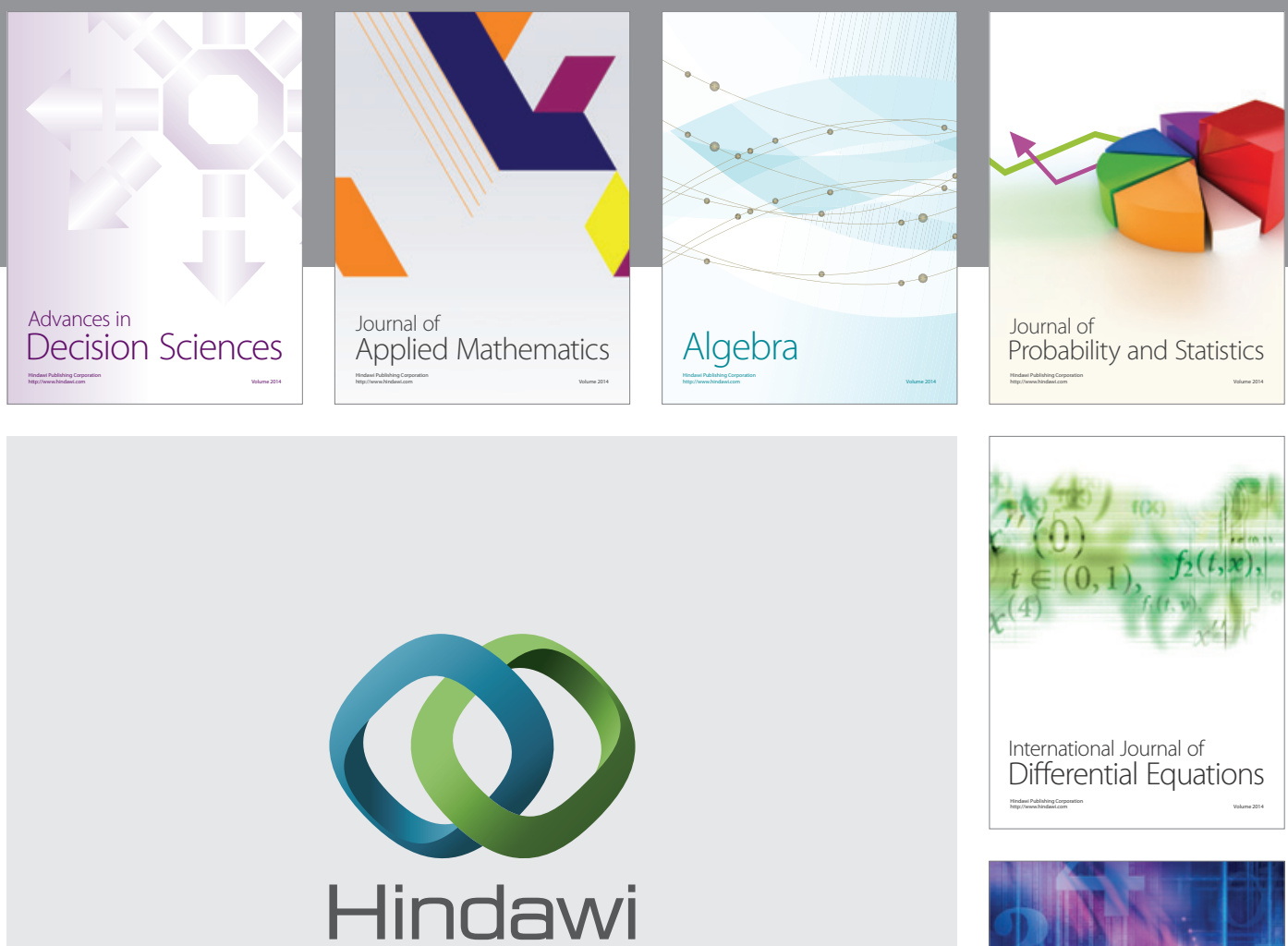

Submit your manuscripts at http://www.hindawi.com
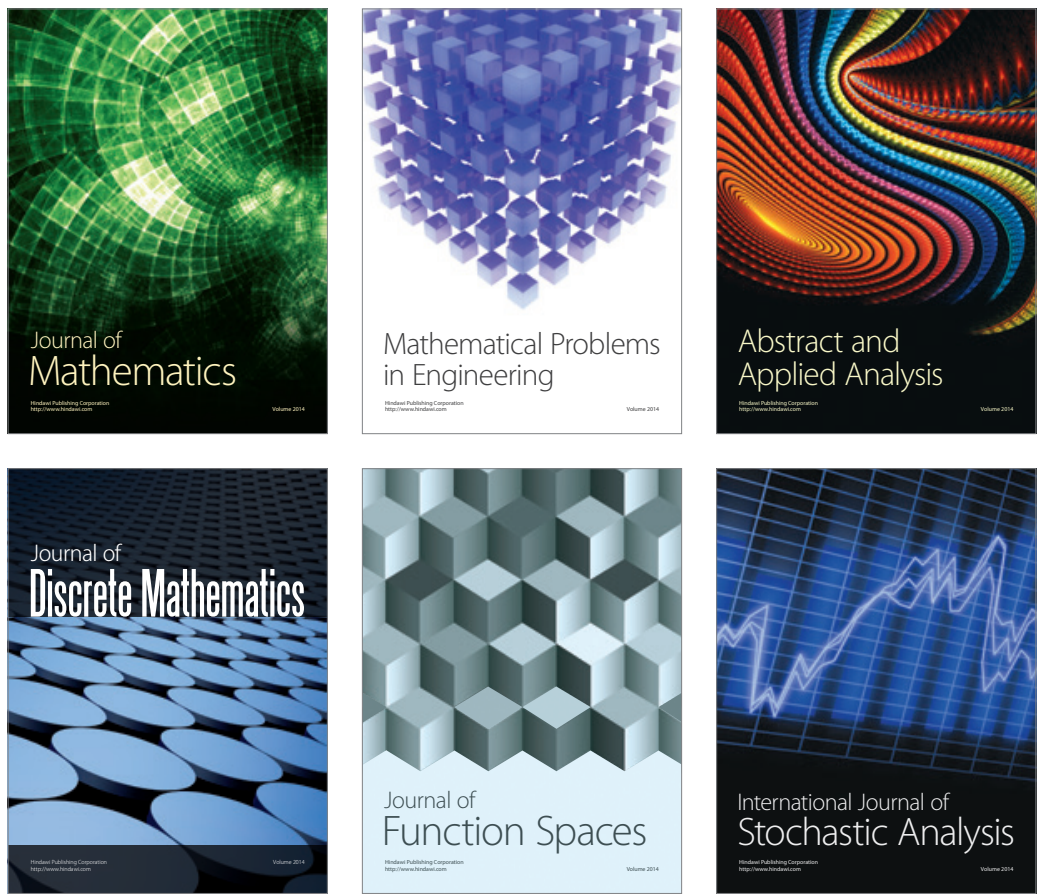

Journal of

Function Spaces

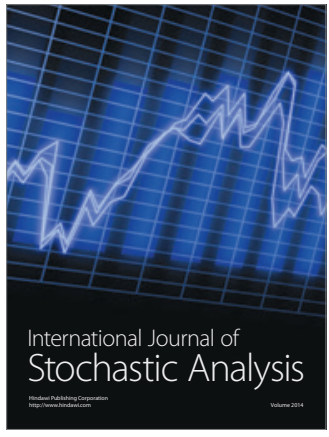

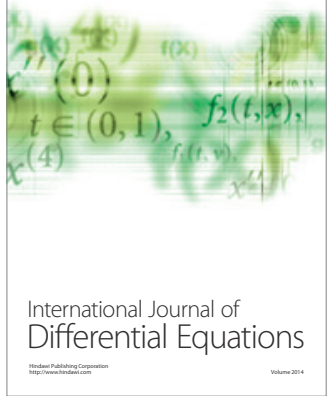
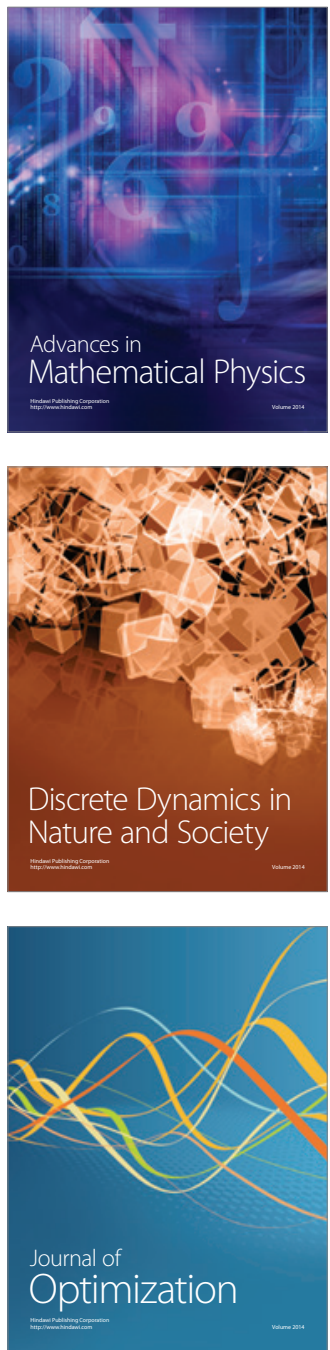